\title{
Article \\ Amended Criteria of Oscillation for Nonlinear Functional Dynamic Equations of Second-Order
}

\author{
Taher S. Hassan 1,2,*, Rami Ahmad El-Nabulsi ${ }^{3,4,5, *}$ and Amir Abdel Menaem 6 \\ 1 Department of Mathematics, Faculty of Science, University of Ha'il, Ha'il 2440, Saudi Arabia \\ 2 Department of Mathematics, Faculty of Science, Mansoura University, Mansoura 35516, Egypt \\ 3 Research Center for Quantum Technology, Faculty of Science, Chiang Mai University, \\ Chiang Mai 50200, Thailand \\ 4 Department of Physics and Materials Science, Faculty of Science, Chiang Mai University, \\ Chiang Mai 50200, Thailand \\ 5 Athens Institute for Education and Research, Mathematics and Physics Divisions, 8 Valaoritou Street, \\ Kolonaki, 10671 Athens, Greece \\ 6 Department of Automated Electrical Systems, Ural Power Engineering Institute, Ural Federal University, \\ 620002 Yekaterinburg, Russia; abdel.menaem@urfu.ru \\ * Correspondence: tshassan@mans.edu.eg (T.S.H.); \\ el-nabulsi@atiner.gr or nabulsiahmadrami@yahoo.fr (R.A.E.-N.)
}

check for updates

Citation: Hassan, T.S.; El-Nabulsi, R.A.; Abdel Menaem, A. Amended Criteria of Oscillation for Nonlinear Functional Dynamic Equations of Second-Order. Mathematics 2021, 9, 1191. https://doi.org/10.3390/ math9111191

Academic Editor: Ignazio Licata

Received: 13 February 2021

Accepted: 19 May 2021

Published: 25 May 2021

Publisher's Note: MDPI stays neutral with regard to jurisdictional claims in published maps and institutional affiliations.

Copyright: (c) 2021 by the authors. Licensee MDPI, Basel, Switzerland. This article is an open access article distributed under the terms and conditions of the Creative Commons Attribution (CC BY) license (https:/ / creativecommons.org/licenses/by/ $4.0 /)$.

\begin{abstract}
In this paper, the sharp Hille-type oscillation criteria are proposed for a class of secondorder nonlinear functional dynamic equations on an arbitrary time scale, by using the technique of Riccati transformation and integral averaging method. The obtained results demonstrate an improvement in Hille-type compared with the results reported in the literature. Some examples are provided to illustrate the significance of the obtained results.
\end{abstract}

Keywords: oscillation behavior; second-order; nonlinear; functional dynamic equation; time scales

\section{Introduction}

The theory of time scales, which has recently received a lot of interest, was proposed by Stefan Hilger in 1988 in order to unite continuous and discrete analysis; see [1]. The theory was introduced in reality to amalgamate continuous and discrete analyses, which are the basic stones in dynamical systems. The theory of differential equations is one of these theories that can be explored and analyzed by means of time scales to their wide implications in real-word systems and processes. Some of these systems include ecosystems, electronic engineering, biomathematics, signal processing, control theory, stochastic biochemical and transport processes, etc. Moreover, several mathematical topics, such as stability analysis, boundary values problems and perturbations techniques are better explored on time scales; see [2-13]. A time scale $\mathbb{T}$ is an arbitrary closed subset of the reals. The forward jump operator $\sigma: \mathbb{T} \rightarrow \mathbb{T}$ is given by

$$
\sigma(\xi)=\inf \{s \in \mathbb{T}: s>\xi\}
$$

where $\inf \phi=\sup \mathbb{T}$, and it is called that $f: \mathbb{T} \rightarrow \mathbb{R}$ is differentiable at $\xi \in \mathbb{T}$ provided

$$
f^{\Delta}(\xi):=\lim _{s \rightarrow \xi} \frac{f(\xi)-f(s)}{\xi-s},
$$

exists when $\sigma(\xi)=\xi$ and when $f$ is continuous at $\xi$ and $\sigma(\xi)>\xi$,

$$
f^{\Delta}(\xi):=\frac{f(\sigma(\xi))-f(\xi)}{\sigma(\xi)-\xi} .
$$


It is notable that when this time scale is equal to the reals or to the integers representing the classical theories of differential and of difference equations, many other interesting time scales exist, and they give rise to many applications (see [14]). This new theory of these so-called "dynamic equations" not only unifies the corresponding theories for the differential equations and difference equations cases, but it also extends these classical cases to cases "in between". That is, we are worthy of considering the $q$-difference equations when $\mathbb{T}=q^{\mathbb{N}_{0}}:=\left\{q^{\lambda}: \lambda \in \mathbb{N}_{0}\right.$ for $\left.q>1\right\}$, which has important applications in quantum theory (see [15]), and various types of time scale such as $\mathbb{T}=h \mathbb{N}, \mathbb{T}=\mathbb{N}^{2}$, and $\mathbb{T}=\mathbb{T}_{n}$, where $\mathbb{T}_{n}$ is the set of the harmonic numbers, can also be considered. For an outstanding introduction to calculus on time scales, see $[1,14,16,17]$.

The qualitative properties analysis of the dynamic equations is vital, not just for further development of the oscillation theory but also for practical reasons. In fact, the study of quasilinear equations has become a crucial area of research thanks to the fact that such equations arise during a variety of phenomena; for more details, see $[18,19]$. Therefore, this work is concerned about the behavior of the oscillatory solutions to the quasilinear functional dynamic equation of second-order

$$
\left(b(\xi)\left|x^{\Delta}(\xi)\right|^{\beta-1} x^{\Delta}(\xi)\right)^{\Delta}+c(\xi)|x(\eta(\xi))|^{\gamma-1} x(\eta(\xi))=0
$$

on an above-unbounded time scale $\mathbb{T}$, where $\xi \in\left[\xi_{0}, \infty\right)_{\mathbb{T}}, \xi_{0} \geq 0, \xi_{0} \in \mathbb{T} ; \beta$ and $\gamma$ are positive real numbers; $b, c \in \mathrm{C}_{\mathrm{rd}}\left(\left[\xi_{0}, \infty\right) \mathbb{T}_{\mathbb{T}}, \mathbb{R}^{+}\right)$such that

$$
\int^{\infty} \frac{\Delta \tau}{b^{\frac{1}{\beta}}(\tau)}=\infty
$$

and $\eta: \mathbb{T} \rightarrow \mathbb{T}$ is a rd-continuous function such that $\lim _{\xi \rightarrow \infty} \eta(\xi)=\infty$.

By a solution of the Equation (4), we mean a nontrivial real-valued function $x \in$ $\mathrm{C}_{\mathrm{rd}}^{1}\left[\xi_{x}, \infty\right)_{\mathbb{T}}, \xi_{x} \in\left[\xi_{0}, \infty\right)_{\mathbb{T}}$ such that $b\left|x^{\Delta}\right|^{\beta-1} x^{\Delta} \in \mathrm{C}_{\mathrm{rd}}^{1}\left[\xi_{x}, \infty\right)_{\mathbb{T}}$ and $x$ satisfies (4) on $\left[\xi_{x}, \infty\right)_{\mathbb{T}}$, where $C_{\text {rd }}$ is the set of right-dense continuous functions. A solution $x$ of (4) is termed oscillatory if it is neither eventually positive nor eventually negative; otherwise, we call it nonoscillatory. The solutions vanishing in some neighborhood of infinity will be excluded from our consideration. In the sequel, all functional inequalities considered are tacitly supposed to hold eventually; that is, they are satisfied for all $\xi$ which are sufficiently large.

It may be noted that, in a particular case, when $\mathbb{T}=\mathbb{R}$, then

$$
\mu(\xi)=0, \eta^{\Delta}(\xi)=\eta^{\prime}(\xi), \int_{a}^{b} \eta(\xi) \Delta \xi=\int_{a}^{b} \eta(\xi) d \xi,
$$

and the Equation (4) becomes the quasilinear differential equation

$$
\left(b(\xi)\left|x^{\prime}(\xi)\right|^{\beta-1} x^{\prime}(\xi)\right)^{\prime}+c(\xi)|x(\eta(\xi))|^{\gamma-1} x(\eta(\xi))=0
$$

The Hille-type oscillation criterion of special cases of Equation (7) is investigated by [20-22], such as Erbe [22] examined the Hille-type oscillation criterion and shown that if

$$
\liminf _{\xi \rightarrow \infty} \xi \int_{\xi}^{\infty} q(\tau) \frac{\eta(\tau)}{\tau} \mathrm{d} \tau>\frac{1}{4}
$$

then all solutions of

$$
x^{\prime \prime}(\xi)+c(\xi) x(\eta(\xi))=0
$$

are oscillatory in which $\eta(\xi) \leq \xi$. 
If $\mathbb{T}=\mathbb{Z}$, then

$$
\mu(\xi)=1, \eta^{\Delta}(\xi)=\Delta \eta(\xi), \int_{a}^{b} \eta(\xi) \Delta \xi=\sum_{\xi=a}^{b-1} \eta(\xi),
$$

and (4) becomes the quasilinear difference equation

$$
\Delta\left(b(\xi)|\Delta x(\xi)|^{\beta-1} \Delta x(\xi)\right)+c(\xi)|x(\eta(\xi))|^{\gamma-1} x(\eta(\xi))=0 .
$$

For further application on other types of time scale, see [14,17].

For Hille-type oscillation criteria of second-order dynamic equations, References [23-27] proposed some Hille-type oscillation criteria to special cases of Equation (7). Recently, Hassan et al. [28] established the following Hille-type oscillation criterion for (4) and showed that if $l>0$ and

$$
\liminf _{\xi \rightarrow \infty} B^{\beta}(\xi) \int_{\sigma(\xi)}^{\infty} \phi(\tau) Q(\tau) \Delta \tau>\frac{\beta^{\beta}}{l \beta^{2}(\beta+1)^{\beta+1}},
$$

then all solutions of Equation (4) oscillate, where

$$
\begin{gathered}
B(\xi):=\int_{\tilde{\xi}_{0}}^{\xi} \frac{\Delta \tau}{b^{\frac{1}{\gamma}}(\tau)} \text { and } l:=\liminf _{\xi \rightarrow \infty} \frac{B(\xi)}{B(\sigma(\xi))} \leq 1, \\
\phi(\xi):= \begin{cases}1, & \eta(\xi) \geq \xi, \\
\left(\frac{B(\eta(\xi))}{B(\xi)}\right)^{\gamma}, & \eta(\xi) \leq \xi,\end{cases}
\end{gathered}
$$

and

$$
Q(\xi):= \begin{cases}c(\xi), & \gamma \geq \beta, \\ c(\xi)\left[\int_{\xi}^{\infty} \phi(\tau) c(\tau) \Delta \tau\right]^{\frac{\beta-\gamma}{\gamma},} & \gamma \leq \beta,\end{cases}
$$

provided the improper integrals involved are convergent. These oscillation criteria for (4) were established in the cases: $\eta(\xi) \leq \xi, \eta(\xi) \geq \xi, \gamma \geq \beta$, and $\gamma \leq \beta$ without the restrictive condition

$$
\int_{\tilde{\xi}_{0}}^{\infty} \eta^{\beta}(\tau) c(\tau) \Delta \tau=\infty
$$

in contrast to [23,24]. We refer the reader to related results [29-42] and the references cited therein.

The object of this paper is to deduce some sharp Hille-type oscillation criteria for (4) in the cases where $\gamma \geq \beta$ and $\gamma \leq \beta$ and for the both cases advanced and delayed dynamic equations. The results which will be proven in this paper present critical improvement to the results in $[23,24,28]$; for more details, see Section 4.

It is notable that the oscillatory solutions are of particular importance in the theory of differential equations due to their particular asymptotic behavior of all solutions of second-order half-linear differential equations. These solutions are particularly important in physics, when damping occurs in nonlinear classical and quantum dynamical systems; see [43-46].

This paper is organized as follows: we state the main results and proofs for Equation (4) in Sections 2 and 3 when $\gamma \geq \beta$ and $\gamma \leq \beta$ respectively, followed by demonstrating examples. Discussions are given in Section 4. 


\section{Oscillation Criteria of (4) when $\gamma \geq \beta$}

In the following, we utilize the notation

$$
\omega(\xi):= \begin{cases}1, & \eta(\xi) \geq \sigma(\xi), \\ \left(\frac{B(\eta(\xi))}{B(\sigma(\xi))}\right)^{\gamma}, & \eta(\xi) \leq \sigma(\xi),\end{cases}
$$

Furthermore, we assume, in the next theorems, that the improper integrals are convergent. Otherwise, we obtain that Equation (4) is oscillatory; see [47].

The next theorem is Hille type to the second-order quasilinear dynamic Equation (4) when $\gamma \geq \beta$.

Theorem 1. Let $0<\beta \leq 1$ and (5) holds. If $l>0$ and

$$
\liminf _{\xi \rightarrow \infty} B^{\beta}(\xi) \int_{\xi}^{\infty} \omega(\tau) c(\tau) \Delta \tau>\frac{\beta^{\beta}}{l^{\beta(1-\beta)}(\beta+1)^{\beta+1}},
$$

then all solutions of Equation (4) oscillate.

Proof. Assume to the contrary that Equation (4) has a nonoscillatory solution $x$ on $\left[\xi_{0}, \infty\right)_{\mathbb{T}}$. Without loss of generality, we let $x(\xi)>0$ and $x(\eta(\xi))>0$ for $\xi \in\left[\xi_{0}, \infty\right)_{\mathbb{T}}$. Define

$$
w(\xi):=\frac{b(\xi)\left|x^{\Delta}(\xi)\right|^{\beta-1} x^{\Delta}(\xi)}{x^{\beta}(\xi)} .
$$

By the rules of product and quotient, we get

$$
\begin{aligned}
w^{\Delta}(\xi) & =\frac{1}{x^{\beta}(\sigma(\xi))}\left[b(\xi)\left|x^{\Delta}(\xi)\right|^{\beta-1} x^{\Delta}(\xi)\right]^{\Delta}+\left(\frac{1}{x^{\beta}(\xi)}\right)^{\Delta} b(\xi)\left|x^{\Delta}(\xi)\right|^{\beta-1} x^{\Delta}(\xi) \\
& =\frac{\left[b(\xi)\left|x^{\Delta}(\xi)\right|^{\beta-1} x^{\Delta}(\xi)\right]^{\Delta}}{x^{\beta}(\sigma(\xi))}-\frac{\left(x^{\beta}(\xi)\right)^{\Delta}}{x^{\beta}(\xi) x^{\beta}(\sigma(\xi))} b(\xi)\left|x^{\Delta}(\xi)\right|^{\beta-1} x^{\Delta}(\xi) .
\end{aligned}
$$

From (4) and (19), we conclude that

$$
w^{\Delta}(\xi)=-\left(\frac{x(\eta(\xi))}{x(\sigma(\xi))}\right)^{\gamma} x^{\gamma-\beta}(\sigma(\xi)) c(\xi)-\frac{\left(x^{\beta}(\xi)\right)^{\Delta}}{x^{\beta}(\sigma(\xi))} w(\xi) .
$$

Since $c \in \mathrm{C}_{\mathrm{rd}}\left(\left[\xi_{0}, \infty\right)_{\mathbb{T}}, \mathbb{R}^{+}\right)$and then

$$
\left(b(\xi)\left|x^{\Delta}(\xi)\right|^{\beta-1} x^{\Delta}(\xi)\right)^{\Delta}<0 \quad \text { for } \xi \geq \xi_{0} .
$$

Hence $x^{\Delta}(\xi)>0$, otherwise, it leads to a contradiction. Thus

$$
x^{\gamma-\beta}(\sigma(\xi)) \geq x^{\gamma-\beta}\left(\sigma\left(\xi_{0}\right)\right)=: k>0 \text { for } \xi \geq \xi_{0} .
$$

Therefore,

$$
w^{\Delta}(\xi) \leq-k\left(\frac{x(\eta(\xi))}{x(\sigma(\xi))}\right)^{\gamma} c(\xi)-\frac{\left(x^{\beta}(\xi)\right)^{\Delta}}{x^{\beta}(\sigma(\xi))} w(\xi) .
$$

It follows from ([28], Theorem 1$)$ that $\frac{x(\xi)}{B(\xi)}$ is strictly decreasing and

$$
x(\xi) \geq b(\xi)\left|x^{\Delta}(\xi)\right|^{\beta-1} x^{\Delta}(\xi) B^{\beta}(\xi) \quad \text { on }\left(\xi_{0}, \infty\right)_{\mathbb{T}} .
$$


Therefore, there exists a $\xi_{1} \in\left(\xi_{0}, \infty\right)_{\mathbb{T}}$ such that for $\xi \in\left[\xi_{1}, \infty\right)_{\mathbb{T}}$,

$$
\left(\frac{x(\eta(\xi))}{x(\sigma(\xi))}\right)^{\gamma} \geq \omega(\xi)
$$

Hence, we obtain, for every $k>0$ and for $\xi \in\left[\xi_{1}, \infty\right)_{\mathbb{T}}$,

$$
w^{\Delta}(\xi) \leq-k \omega(\xi) c(\xi)-\frac{\left(x^{\beta}(\xi)\right)^{\Delta}}{x^{\beta}(\sigma(\xi))} w(\xi) .
$$

Using Pötzsche chain rule (see [14], Theorem 1.90), we get

$$
\left(x^{\beta}(\xi)\right)^{\Delta}=\beta\left(\int_{0}^{1}[(1-h) x(\xi)+h x(\sigma(\xi))]^{\beta-1} \mathrm{~d} h\right) x^{\Delta}(\xi) \geq \beta x^{\beta-1}(\sigma(\xi)) x^{\Delta}(\xi) .
$$

Hence, by virtue of

$$
\begin{aligned}
\frac{x^{\Delta}(\xi)}{x(\sigma(\xi))} & =\frac{1}{x(\sigma(\xi))}\left[\frac{b(\xi)\left(x^{\Delta}(\xi)\right)^{\beta}}{b(\xi)}\right]^{\frac{1}{\beta}} \\
& \geq \frac{1}{x(\sigma(\xi))}\left[\frac{b(\sigma(\xi))\left(x^{\Delta}(\sigma(\xi))\right)^{\beta}}{b(\xi)}\right]^{\frac{1}{\beta}}=b^{-\frac{1}{\beta}(\xi) w^{\frac{1}{\beta}}(\sigma(\xi))}
\end{aligned}
$$

we deduce that

$$
\begin{aligned}
w^{\Delta}(\xi) & \leq-k \omega(\xi) c(\xi)-\beta \frac{x^{\Delta}(\xi)}{x(\sigma(\xi))} w(\xi) \\
& \leq-k \omega(\xi) c(\xi)-\beta b^{-\frac{1}{\beta}}(\xi) w^{\frac{1}{\beta}}(\sigma(\xi)) w(\xi),
\end{aligned}
$$

which yields $w^{\Delta}<0$. Integrating (21) from $\xi$ to $v$, we achieve

$$
w(v)-w(\xi) \leq-k \int_{\xi}^{v} \omega(\tau) c(\tau) \Delta \tau-\beta \int_{\xi}^{v} b^{-\frac{1}{\beta}}(\tau) w^{\frac{1}{\beta}}(\sigma(\tau)) w(\tau) \Delta \tau,
$$

and thus

$$
-w(\xi) \leq-k \int_{\xi}^{\infty} \omega(\tau) c(\tau) \Delta \tau-\beta \int_{\xi}^{\infty} b^{-\frac{1}{\beta}}(\tau) w^{\frac{1}{\beta}}(\sigma(\tau)) w(\tau) \Delta \tau
$$

due to the fact that $w>0$. Multiplying both sides of (22) by $B^{\beta}(\xi)$, we have

$$
\begin{aligned}
-B^{\beta}(\xi) w(\xi) \leq & -k B^{\beta}(\xi) \int_{\xi}^{\infty} \omega(\tau) c(\tau) \Delta \tau \\
& -\beta B^{\beta}(\xi) \int_{\xi}^{\infty} b^{-\frac{1}{\beta}}(\tau) w^{\frac{1}{\beta}}(\sigma(\tau)) w(\tau) \Delta \tau \\
= & -k B^{\beta}(\xi) \int_{\xi}^{\infty} \omega(\tau) c(\tau) \Delta \tau \\
& -\beta B^{\beta}(\xi) \int_{\xi}^{\infty} \frac{b^{-\frac{1}{\beta}}(\tau)}{B^{\beta}(\tau) B(\sigma(\tau))} B^{\beta}(\tau) w(\tau) B(\sigma(\tau)) w^{\frac{1}{\beta}}(\sigma(\tau)) \Delta \tau .
\end{aligned}
$$

Now, for any $\varepsilon>0$, there exists a $\xi_{2} \in\left[\xi_{1}, \infty\right)_{\mathbb{T}}$ such that, for $\xi \in\left[\xi_{2}, \infty\right)_{\mathbb{T}}$,

$$
\frac{B(\xi)}{B(\sigma(\xi))} \geq l-\varepsilon \quad \text { and } \quad B^{\beta}(\xi) w(\xi) \geq b_{*}-\varepsilon
$$


where

$$
b_{*}:=\liminf _{\xi \rightarrow \infty} B^{\beta}(\xi) w(\xi), \quad 0 \leq b_{*} \leq 1
$$

due to (19) and (20). Therefore,

$$
\begin{aligned}
-B^{\beta}(\xi) w(\xi) \leq & -k B^{\beta}(\xi) \int_{\xi}^{\infty} \omega(\tau) c(\tau) \Delta \tau \\
& -\left(b_{*}-\varepsilon\right)^{1+\frac{1}{\beta}} B^{\beta}(\xi) \int_{\xi}^{\infty} \frac{\beta B^{\Delta}(\tau)}{B(\tau) B^{\beta}(\sigma(\tau))}\left(\frac{B(\tau)}{B(\sigma(\tau))}\right)^{1-\beta} \Delta \tau \\
\leq & -k B^{\beta}(\xi) \int_{\xi}^{\infty} \omega(\tau) c(\tau) \Delta \tau \\
& -(l-\varepsilon)^{1-\beta}\left(b_{*}-\varepsilon\right)^{1+\frac{1}{\beta}} \int_{\tilde{\xi}}^{\infty} \frac{\beta B^{\Delta}(\tau)}{B(\tau) B^{\beta}(\sigma(\tau))} \Delta \tau .
\end{aligned}
$$

Thus, Pötzsche chain rule implies that

$$
\left(B^{\beta}(\tau)\right)^{\Delta}=\beta \int_{0}^{1}[(1-h) B(\tau)+h B(\sigma(\tau))]^{\beta-1} \mathrm{~d} h B^{\Delta}(\tau) \leq \beta B^{\beta-1}(\sigma(\tau)) B^{\Delta}(\tau) .
$$

It follows now from (24) and the quotient rule that

$$
\left(B^{\beta}(\tau)\right)^{\Delta}=\beta \int_{0}^{1}[(1-h) B(\tau)+h B(\sigma(\tau))]^{\beta-1} \mathrm{~d} h B^{\Delta}(\tau) \leq \beta B^{\beta-1}(\tau) B^{\Delta}(\tau)
$$

and so

$$
\left(\frac{-1}{B^{\beta}(\tau)}\right)^{\Delta}=\frac{\left(B^{\beta}(\tau)\right)^{\Delta}}{B^{\beta}(\tau) B^{\beta}(\sigma(\tau))} \leq \frac{\beta B^{\Delta}(\tau)}{B(\tau) B^{\beta}(\sigma(\tau))} .
$$

From (23), we have

$$
\begin{aligned}
-B^{\beta}(\xi) w(\xi) \leq & -k B^{\beta}(\xi) \int_{\xi}^{\infty} \omega(\tau) c(\tau) \Delta \tau \\
& -\left(b_{*}-\varepsilon\right)^{1+\frac{1}{\beta}} B^{\beta}(\xi) \int_{\xi}^{\infty}\left(\frac{-1}{B^{\beta}(\tau)}\right)^{\Delta}\left(\frac{B(\tau)}{B(\sigma(\tau))}\right)^{1-\beta} \Delta \tau \\
= & -k B^{\beta}(\xi) \int_{\xi}^{\infty} \omega(\tau) c(\tau) \Delta \tau-(l-\varepsilon)^{1-\beta}\left(b_{*}-\varepsilon\right)^{1+\frac{1}{\beta}},
\end{aligned}
$$

which yields that

$$
k B^{\beta}(\xi) \int_{\xi}^{\infty} \omega(\tau) c(\tau) \Delta \tau \leq B^{\beta}(\xi) w(\xi)-(l-\varepsilon)^{1-\beta}\left(b_{*}-\varepsilon\right)^{1+\frac{1}{\beta}} .
$$

Taking the lim inf of both sides as $\xi \rightarrow \infty$, we obtain

$$
\liminf _{\tilde{\xi} \rightarrow \infty} k B^{\beta}(\xi) \int_{\tilde{\xi}}^{\infty} \omega(\tau) c(\tau) \Delta \tau \leq b_{*}-(l-\varepsilon)^{1-\beta}\left(b_{*}-\varepsilon\right)^{1+\frac{1}{\beta}} .
$$

By dint of the facts that $k$ and $\varepsilon>0$ are arbitrary, we arrive at

$$
\liminf _{\xi \rightarrow \infty} B^{\beta}(\xi) \int_{\xi}^{\infty} \omega(\tau) c(\tau) \Delta \tau \leq b_{*}-l^{1-\beta} b_{*}^{1+\frac{1}{\beta}}
$$

Let

$$
B=l^{\beta-1}, \quad A=1, \quad \text { and } \quad u=b_{*} .
$$


By the inequality

$$
A u-B u^{1+\frac{1}{\beta}} \leq \frac{\beta^{\beta}}{(\beta+1)^{\beta+1}} \frac{A^{\beta+1}}{B^{\beta}}, \quad A, B>0
$$

Hence,

$$
\liminf _{\xi \rightarrow \infty} B^{\beta}(\xi) \int_{\xi}^{\infty} \omega(\tau) c(\tau) \Delta \tau \leq \frac{\beta^{\beta}}{l^{\beta(1-\beta)}(\beta+1)^{\beta+1}},
$$

which gives us the contradiction with (18). This completes the proof.

Example 1. For $\xi \in\left[\xi_{0}, \infty\right)_{\mathbb{T}}$, consider a second-order quasilinear functional dynamic equation

$$
\left[\xi^{1 / \beta}\left|x^{\Delta}(\xi)\right|^{\beta-1} x^{\Delta}(\xi)\right]^{\Delta}+\frac{\beta \gamma}{\xi \omega(\xi) B(\xi) B^{\beta}(\sigma(\xi))}|x(\eta(\xi))|^{\gamma-1} x(\eta(\xi))=0
$$

where $0<\beta \leq 1$. It is easy to see that (5) is satisfied. Additionally,

$$
\begin{aligned}
\liminf _{\xi \rightarrow \infty} B^{\beta}(\xi) \int_{\xi}^{\infty} \omega(\tau) c(\tau) \Delta \tau & =\gamma \liminf _{\xi \rightarrow \infty} B^{\beta}(\xi) \int_{\xi}^{\infty} \frac{\beta}{\tau B(\tau) B^{\beta}(\sigma(\tau))} \Delta \tau \\
& \geq \gamma \liminf _{\xi \rightarrow \infty} B^{\beta}(\xi) \int_{\xi}^{\infty}\left(\frac{-1}{B^{\beta}(\tau)}\right)^{\Delta} \Delta \tau=\gamma
\end{aligned}
$$

Then, Theorem 1 implies that every solution of (26) is oscillatory if

$$
0<\beta \leq 1 \text { and } \gamma>\frac{\beta^{\beta}}{l^{\beta(1-\beta)}(\beta+1)^{\beta+1}} .
$$

Theorem 2. Let $\beta \geq 1$ and (5) holds. If $l>0$ and

$$
\liminf _{\xi \rightarrow \infty} B^{\beta}(\xi) \int_{\xi}^{\infty} \phi(\tau) c(\tau) \Delta \tau>\frac{\beta^{\beta}}{l^{\beta(\beta-1)}(\beta+1)^{\beta+1}},
$$

then all solutions of Equation (4) oscillate.

Proof. Assume to the contrary that Equation (4) has a nonoscillatory solution $x$ on $\left[\xi_{0}, \infty\right)_{\mathbb{T}}$. Without loss of generality, we let $x(\xi)>0$ and $x(\eta(\xi))>0$ on $\left[\xi_{0}, \infty\right)_{\mathbb{T}}$. It is easy to achieve that (see [28], Theorem 1)

$$
\begin{aligned}
w^{\Delta}(\xi) & \leq-k \phi(\xi) c(\xi)-\beta \frac{x^{\Delta}(\xi)}{x(\xi)} w(\sigma(\xi)) \\
& =-k \phi(\xi) c(\xi)-\beta b^{-\frac{1}{\beta}}(\xi) w^{\frac{1}{\beta}}(\xi) w(\sigma(\xi))
\end{aligned}
$$

where $k>0$ is an arbitrary constant, $\phi(\xi)$ and $w(\xi)$ are defined by (14) and (19) respectively. Integrating (28) from $\xi$ to $v$, we get

$$
w(v)-w(\xi) \leq-k \int_{\xi}^{v} \phi(\tau) c(\tau) \Delta \tau-\beta \int_{\xi}^{v} b^{-\frac{1}{\beta}}(\tau) w^{\frac{1}{\beta}}(\tau) w(\sigma(\tau)) \Delta \tau .
$$

Taking into account that $w>0$ and passing to the limit as $v \rightarrow \infty$, we obtain

$$
-w(\xi) \leq-k \int_{\xi}^{\infty} \phi(\tau) c(\tau) \Delta \tau-\beta \int_{\xi}^{\infty} b^{-\frac{1}{\beta}}(\tau) w^{\frac{1}{\beta}}(\tau) w(\sigma(\tau)) \Delta \tau .
$$


Multiplying both sides of (29) by $B^{\beta}(\xi)$, we have

$$
\begin{aligned}
-B^{\beta}(\xi) w(\xi) \leq & -k B^{\beta}(\xi) \int_{\xi}^{\infty} \phi(\tau) c(\tau) \Delta \tau \\
& -\beta B^{\beta}(\xi) \int_{\xi}^{\infty} b^{-\frac{1}{\beta}}(\tau) w^{\frac{1}{\beta}}(\tau) w(\sigma(\tau)) \Delta \tau \\
= & -k B^{\beta}(\xi) \int_{\xi}^{\infty} \phi(\tau) c(\tau) \Delta \tau \\
& -\beta B^{\beta}(\xi) \int_{\xi}^{\infty} \frac{b^{-\frac{1}{\beta}}(\tau)}{B(\tau) B^{\beta}(\sigma(\tau))} B(\tau) w^{\frac{1}{\beta}}(\tau) B^{\beta}(\sigma(\tau)) w(\sigma(\tau)) \Delta \tau .
\end{aligned}
$$

It follows from (23) that, for any $\varepsilon>0$,

$$
\begin{aligned}
-B^{\beta}(\xi) w(\xi) \leq & -k B^{\beta}(\xi) \int_{\xi}^{\infty} \phi(\tau) c(\tau) \Delta \tau \\
& -\left(b_{*}-\varepsilon\right)^{1+\frac{1}{\beta}} B^{\beta}(\xi) \int_{\xi}^{\infty} \frac{\beta B^{\Delta}(\tau)}{B^{\beta}(\tau) B(\sigma(\tau))}\left(\frac{B(\tau)}{B(\sigma(\tau))}\right)^{\beta-1} \Delta \tau \\
\leq & -k B^{\beta}(\xi) \int_{\xi}^{\infty} \phi(\tau) c(\tau) \Delta \tau \\
- & (l-\varepsilon)^{\beta-1}\left(b_{*}-\varepsilon\right)^{1+\frac{1}{\beta}} B^{\beta}(\xi) \int_{\xi}^{\infty} \frac{\beta B^{\Delta}(\tau)}{B^{\beta}(\tau) B(\sigma(\tau))} \Delta \tau .
\end{aligned}
$$

On the other hand, by Pötzsche chain rule, we conclude that

$$
\left(B^{\beta}(\tau)\right)^{\Delta}=\beta \int_{0}^{1}[(1-h) B(\tau)+h B(\sigma(\tau))]^{\beta-1} \mathrm{~d} h B^{\Delta}(\tau) \leq \beta B^{\beta-1}(\sigma(\tau)) B^{\Delta}(\tau) .
$$

It follows now from (31) and the quotient rule that

$$
\left(\frac{-1}{B^{\beta}(\tau)}\right)^{\Delta}=\frac{\left(B^{\beta}(\tau)\right)^{\Delta}}{B^{\beta}(\tau) B^{\beta}(\sigma(\tau))} \leq \frac{\beta B^{\Delta}(\tau)}{B^{\beta}(\tau) B(\sigma(\tau))} .
$$

Using (32) in (30), we deduce that

$$
\begin{aligned}
-B^{\beta}(\xi) w(\xi) \leq & -k B^{\beta}(\xi) \int_{\xi}^{\infty} \phi(\tau) c(\tau) \Delta \tau \\
& -(l-\varepsilon)^{\beta-1}\left(b_{*}-\varepsilon\right)^{1+\frac{1}{\beta}} B^{\beta}(\xi) \int_{\tilde{\xi}}^{\infty}\left(\frac{-1}{B^{\beta}(\tau)}\right)^{\Delta} \Delta \tau \\
= & -k B^{\beta}(\xi) \int_{\xi}^{\infty} \phi(\tau) c(\tau) \Delta \tau-(l-\varepsilon)^{\beta-1}\left(b_{*}-\varepsilon\right)^{1+\frac{1}{\beta}},
\end{aligned}
$$

which yields

$$
k B^{\beta}(\xi) \int_{\xi}^{\infty} \phi(\tau) c(\tau) \Delta \tau \leq B^{\beta}(\xi) w(\xi)-(l-\varepsilon)^{\beta-1}\left(b_{*}-\varepsilon\right)^{1+\frac{1}{\beta}} .
$$

Taking the $\lim \inf$ as $\xi \rightarrow \infty$, we conclude that

$$
\liminf _{\xi \rightarrow \infty} k B^{\beta}(\xi) \int_{\xi}^{\infty} \phi(\tau) c(\tau) \Delta \tau \leq b_{*}-(l-\varepsilon)^{\beta-1}\left(b_{*}-\varepsilon\right)^{1+\frac{1}{\beta}} .
$$

Since $k$ and $\varepsilon>0$ are arbitrary, we achieve

$$
\liminf _{\xi \rightarrow \infty} B^{\beta}(\xi) \int_{\tilde{\zeta}}^{\infty} \phi(\tau) c(\tau) \Delta \tau \leq b_{*}-l^{\beta-1} b_{*}^{1+\frac{1}{\beta}} .
$$


Letting $A=1, B=l^{1-\beta}$, and $u=b_{*}$, and by inequality (25), we arrive at

$$
\liminf _{\xi \rightarrow \infty} B^{\beta}(\xi) \int_{\xi}^{\infty} \phi(\tau) c(\tau) \Delta \tau \leq \frac{\beta^{\beta}}{l \beta(\beta-1)(\beta+1)^{\beta+1}},
$$

which gives us the contradiction with (27). This completes the proof.

Example 2. For $\xi \in\left[\xi_{0}, \infty\right)_{\mathbb{T}}$, consider a second order quasilinear functional dynamic equation

$$
\left[\xi^{1 / \beta}\left|x^{\Delta}(\xi)\right|^{\beta-1} x^{\Delta}(\xi)\right]^{\Delta}+\frac{\beta \gamma}{\xi \omega(\xi) B^{\beta}(\xi) B(\sigma(\xi))}|x(\eta(\xi))|^{\gamma-1} x(\eta(\xi))=0
$$

where $\beta \geq 1$. It is easy to see that (5) is satisfied. Additionally

$$
\begin{aligned}
\liminf _{\xi \rightarrow \infty} B^{\beta}(\xi) \int_{\xi}^{\infty} \phi(\tau) c(\tau) \Delta \tau & =\gamma \liminf _{\xi \rightarrow \infty} B^{\beta}(\xi) \int_{\xi}^{\infty} \frac{\beta}{\tau B^{\beta}(\tau) B(\sigma(\tau))} \Delta \tau \\
& \geq \gamma \liminf _{\xi \rightarrow \infty} B^{\beta}(\xi) \int_{\xi}^{\infty}\left(\frac{-1}{B^{\beta}(\tau)}\right)^{\Delta} \Delta \tau=\gamma .
\end{aligned}
$$

Hence, Theorem 2 implies that every solution of (33) is oscillatory if

$$
\beta \geq 1 \text { and } \gamma>\frac{\beta^{\beta}}{l^{\beta(\beta-1)}(\beta+1)^{\beta+1}} .
$$

\section{Oscillation Criteria of (4) when $\gamma \leq \beta$}

From the same, we can easily prove the following oscillation criterion when $\gamma \leq \beta$.

Theorem 3. Let $0<\beta \leq 1$ and (5) holds. If $l>0$ and

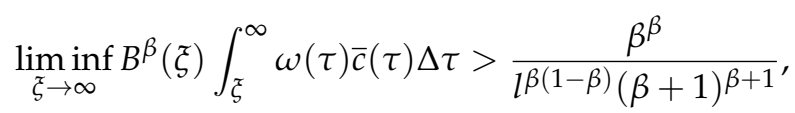

where

$$
\bar{c}(\xi):=c(\xi)\left[\int_{\xi}^{\infty} \phi(\tau) c(\tau) \Delta \tau\right]^{\frac{\beta}{\gamma}-1},
$$

then every solution of (4) is oscillatory.

Theorem 4. Let $\beta \geq 1$, and (5) holds. If $l>0$ and

$$
\liminf _{\tilde{\zeta} \rightarrow \infty} B^{\beta}(\xi) \int_{\tilde{\zeta}}^{\infty} \phi(\tau) \bar{c}(\tau) \Delta \tau>\frac{\beta^{\beta}}{l^{\beta(\beta-1)}(\beta+1)^{\beta+1}},
$$

then every solution of (4) is oscillatory.

\section{Discussions}

(1) In the present work, we established some new improved Hille-type criteria, which can be applied to (4) in the cases where $\gamma \geq \beta$ and $\gamma \leq \beta$ and for both advanced and delayed cases without the restrictive condition in contrast to [23,24].

(2) The results in this paper are correct for various species of time scales, e.g., $\mathbb{T}=\mathbb{R}$, $\mathbb{T}=\mathbb{Z}, \mathbb{T}=h \mathbb{Z}$ with $h>0, \mathbb{T}=q^{\mathbb{N}_{0}}, q>1$ and $\mathbb{T}=\mathbb{N}_{0}^{2}$, etc. (see [14]).

(3) It is easy to see the obtained results have extended related contributions to the differential equations of second-order, for instance conditions (18) and (27) reduces to (8) in the case where $\mathbb{T}=\mathbb{R}, \gamma=\beta=1, b(\xi)=1$, and $\eta(\xi) \leq \xi$. 
(4) Regarding dynamic equations on time scales, the oscillation criteria here are an important improvement compared to the literature outcomes. In particular, our results improve those reported in [28]; see the following details. Let $\beta \geq 1$. By dint of

$$
B^{\beta}(\xi) \int_{\xi}^{\infty} \phi(\tau) Q(\tau) \Delta \tau \geq B^{\beta}(\xi) \int_{\sigma(\xi)}^{\infty} \phi(\tau) Q(\tau) \Delta \tau
$$

and

$$
\frac{\beta^{\beta}}{l \beta(\beta-1)(\beta+1)^{\beta+1}}<\frac{\beta^{\beta}}{l \beta^{2}(\beta+1)^{\beta+1}} \text { for } 0<l<1,
$$

Theorems 2 and 3 improve ([28], Theorems 4 and 8) (conditions (27) and (34) improves (12)).

(5) It would be of interest to establish Hille-type oscillation criteria of (4) assuming that

$$
\int_{\tilde{\xi}_{0}}^{\infty} b^{-\frac{1}{\beta}}(\tau) \Delta \tau<\infty
$$

It would be nice to find work devoted to numerical analysis and real-world applications.

Author Contributions: Formal analysis, T.S.H. and R.A.E.-N.; Investigation, A.A.M.; Supervision, R.A.E.-N. All authors have read and agreed to the published version of the manuscript.

Funding: This research received no external funding.

Institutional Review Board Statement: Not applicable.

Informed Consent Statement: Not applicable.

Data Availability Statement: Not applicable.

Acknowledgments: The authors would like to thank anonymous referees for their careful reading of the entire manuscript, which helped significantly improve this paper's quality. This work was supported by Research Deanship of Hail University under grant No. 0150396.

Conflicts of Interest: The authors declare no conflict of interest.

\section{References}

1. Hilger, S. Analysis on measure chains a unified approach to continuous and discrete calculus. Results Math. 1990, 18, 18-56. [CrossRef]

2. Agarwal, R.P.; Bohner, M. Quadratic functionals for second order matrix equations on time scales. Nonlinear Anal. 1998, 33, 675-692. [CrossRef]

3. Agarwal, R.P.; Bohner, M. Basic calculus on time scales and some of its applications. Results Math. 1999, 35, 3-22. [CrossRef]

4. Bel, G.; Zilman, A.; Kolomeisky, A.B. Different time scales in dynamic systems with multiple outcomes. J. Chem. Phys. 2020, 153, 054107. [CrossRef] [PubMed]

5. Bramburger, J.J.; Dylewsky, D.; Nathan Kutz, J. Sparse identification of slow time scale dynamics. Phys. Rev. 2020, E102, 022204.

6. Akhmet, M.U.; Turan, M. Differential equations on variable time scales. Nonlinear Anal. 2009, 70, 1175-1192. [CrossRef]

7. Akhmet, M.U.; Turan, M. The differential equations on time scales through impulsive differential equations. Nonlinear Anal. 2006, 65, 2043-2060. [CrossRef]

8. Laksmikantham, V.; Sivasundaram, S.; Kaymakcalan, B. Dynamical systems on measure chains. In Mathematics and Its Applications; Kluwer Academic: Dordrecht, The Netherlands, 1996; Volume 370.

9. Nwaeze, E.R.; Torres, D.F.M. Chain rules and inequalities for the BHT fractional calculus on arbitrary time scales. Arab. J. Math. 2017, 6, 13-20. [CrossRef]

10. Zhao, D.; Li, T. On conformable delta fractional calculus on time scales. J. Math. Comput. Sci. 2016, 16, 324-335. [CrossRef]

11. Anderson, D.R.; Ulness, D.J. Newly defined conformable derivatives. Adv. Dyn. Syst. Appl. 2015, 10, $109-137$.

12. Malinowska, A.B.; Martins, N.; Torres, D.F.M. Transversality conditions for infinite horizon variational problems on time scales. Optim. Lett. 2011, 5, 41-53. [CrossRef]

13. Malinowska, A.B.; Torres, D.F.M. Leitmann's direct method of optimization for absolute extrema of certain problems of the calculus of variations on time scales. Appl. Math. Comput. 2010, 217, 1158-1162. [CrossRef]

14. Bohner, M.; Peterson, A. Dynamic Equations on Time Scales: An Introduction with Applications; Birkhäuser: Boston, MA, USA, 2001.

15. Kac, V.; Chueng, P. Quantum Calculus; Universitext, Springer: Berlin, Germany, 2002. 
16. Agarwal, R.P.; Bohner, M.; O’Regan, D.; Peterson, A. Dynamic equations on time scales: A survey. J. Comput. Appl. Math. 2002, 141, 1-26. [CrossRef]

17. Bohner, M.; Peterson, A. Advances in Dynamic Equations on Time Scales; Birkhäuser: Boston, MA, USA, 2003.

18. Došly, O.; Řehák, P. Half-Linear Differential Equations; Elsevier: Amsterdam, The Netherlands, 2005.

19. Agarwal, R.P.; Grace, S.R.; O’Regan, D. Oscillation Theory for Second Order Linear, Half-Linear Superlinear and Sublinear Dynamic Equations; Kluwer Academic Publishers: Dordrecht, The Netherlands, 2002.

20. Hille, E. Non-oscillation theorems. Trans. Am. Math. Soc. 1948, 64, 234-252. [CrossRef]

21. Wong, J.S. Second order oscillation with retarded arguments. In Ordinary Differential Equations; Academic Press: New York, NY, USA; London, UK, 1972; pp. 581-596.

22. Erbe, L. Oscillation criteria for second order quasilinear delay equations. Can. Math. Bull. 1973, 16, 49-56. [CrossRef]

23. Erbe, L.; Hassan, T.S.; Peterson, A.; Saker, S.H. Oscillation criteria for half-linear delay dynamic equations on time scales. Quasilinear Dynam. Sys. Th. 2009, 9, 51-68.

24. Erbe, L.; Hassan, T.S.; Peterson, A.; Saker, S.H. Oscillation criteria for sublinear half-linear delay dynamic equations on time scales. Int. J. Differ. Equ. 2008, 3, 227-245.

25. Bohner, M.; Hassan, T.S.; Li, T. Fite-Hille-Wintner-type oscillation criteria for second-order half-linear dynamic equations with deviating arguments. Indag. Math. 2018, 29, 548-560. [CrossRef]

26. Karpuz, B. Hille-Nehari theorems for dynamic equations with a time scale independent critical constant. Appl. Math. Comput. 2019, 346, 336-351. [CrossRef]

27. Hassan, T.S.; Agarwal, R.P.; Mohammed, W. Oscillation criteria for third-order functional half-linear dynamic equations. Adv. Differ. Equ. 2017, 2017, 111. [CrossRef]

28. Hassan, T.S.; Sun, Y.; Abdel Menaem, A. Improved oscillation results for functional quasilinear dynamic equations of second order. Mathematics 2020, 8, 1897. [CrossRef]

29. Zhang, Q.; Gao, L.; Wang, L. Oscillation of second-order quasilinear delay dynamic equations on time scales. Comput. Math. Appl. 2011, 61, 2342-2348. [CrossRef]

30. Chatzarakis, G.E.; Moaaz, O.; Li, T.; Qaraad, B. Oscillation theorems for quasilinear second-order differential equations with an advanced argument. Adv. Differ. Equ. 2020, 2020, 160. [CrossRef]

31. Baculikova, B. Oscillation of second-order quasilinear noncanonical differential equations with deviating argument. Appl. Math. Lett. 2019, 91, 68-75. [CrossRef]

32. Grace, S.R.; Bohner, M.; Agarwal, R.P. On the oscillation of second-order half-linear dynamic equations. J. Differ. Equ. Appl. 2009, 15, 451-460. [CrossRef]

33. Moaaz, O.; El-Nabulsi, R.A.; Muhsin, W.; Bazighifan, O. Improved Oscillation Criteria for 2nd-Order Neutral Differential Equations with Distributed Deviating Arguments. Mathematics 2020, 8, 849. [CrossRef]

34. Džurina, J.; Jadlovská, I. A sharp oscillation result for second-order half-linear noncanonical delay differential equations. Electron. J. Qual. Theory Differ. Equ. 2020, 46, 1-14. [CrossRef]

35. Nehari, Z. Oscillation criteria for second-order linear differential equations. Trans. Am. Math. Soc. 1957, 85, 428-445. [CrossRef]

36. Ohriska, J. Oscillation of second order delay and ordinary differential equations. Czech. Math. J. 1984, 34, 107-112. [CrossRef]

37. Ǩehak, P. New results on critical oscillation constants depending on a graininess. Dyn. Syst. Appl. 2010, 19, 271-288.

38. Sun, S.; Han, Z.; Zhao, P.; Zhang, C. Oscillation for a class of second-order Emden-Fowler delay dynamic equations on time scales. Adv. Differ. Equ. 2010, 2010, 642356. [CrossRef]

39. Wintner, A. On the nonexistence of conjugate points. Am. J. Math. 1951, 73, 368-380. [CrossRef]

40. Sun, Y.; Hassan, T.S. Oscillation criteria for functional dynamic equations with nonlinearities given by Riemann-Stieltjes integral. Abstr. Appl. Anal. 2014, 2014, 697526. [CrossRef]

41. Saker, S.H. Oscillation criteria of second-order half-linear dynamic equations on time scales. J. Comput. Appl. Math. 2005, 177, 375-387. [CrossRef]

42. Zhang, C.; Agarwal, R.P.; Bohner, M.; Li, T. Properties of higher-order half-linear functional differential equations with noncanonical operators. Adv. Differ. Equ. 2013, 2013, 54. [CrossRef]

43. Shevelo, N.V. Oscillation of Solutions of Differential Equations with Retarded Argument; Naukova Dumka: Kyiv, Ukrania, 1978.

44. Agarwal, R.P.; Berezansky, L.; Braverman, E.; Domoshnitsky, A. Nonoscillation Theory of Functional Differential Equations with Applications; Springer: New York, NY, USA, 2012.

45. Baculikova, B. Oscillatory behavior o the second order functional differential equations. Appl. Math. Lett. 2017, 72, 35-41. [CrossRef]

46. El-Nabulsi, R.A. A periodic functional approach to the calculus of variations and the problem of time-dependent damped harmonic oscillators. Appl. Math. Lett. 2011, 24, 1647-1653.

47. Fite, W.B. Concerning the zeros of the solutions of certain differential equations. Trans. Am. Math. Soc. 1918, 19, 341-352. [CrossRef] 